\title{
Redox regulation of fertilisation and the spermatogenic process
}

\author{
Junichi Fujii ${ }^{1,2,3}$ and Satoshi Tsunoda ${ }^{1}$
}

Oxidative stress is one of the major causes of male infertility; it damages spermatogenic cells, the spermatogenic process and sperm function. Recent advances in redox biology have revealed the signalling role of reactive oxygen species (ROS) that are generated by cells. While highly reactive oxidants, such as the hydroxyl radical, exert largely deleterious effects, hydrogen peroxide can feasibly serve as a signal mediator because it is moderately reactive and membrane permeable and because it can oxidize only limited numbers of functional groups of biological molecules. The amino acid side chain most sensitive to oxidation is cysteine sulphydryl, which is commonly involved in the catalysis of some enzymes. Although the reactivity of cysteine sulphhydryl is not very high in ordinary proteins, some phosphatases possess a highly reactive sulphydryl group at their catalytic centre and are thereby oxidatively inactivated by transiently elevated hydrogen peroxide levels after extracellular stimuli and under certain environmental conditions. Peroxiredoxins, in turn, show moderate hydrogen peroxide-reducing activity, and their role in the modulation of ROS-mediated signal transduction in ordinary cells, mediated by protecting phosphatases from oxidative inactivation, has attracted much attention. Although knowledge of the signalling role of ROS in the male reproductive system is limited at present, its significance is becoming a focal issue. Here, we present a review of the emerging signalling role of hydrogen peroxide in testes.

Asian Journal of Andrology (2011) 13,420-423; doi:10.1038/aja.2011.10; published online 4 April 2011

Keywords: hydrogen peroxide; oxidative stress; peroxiredoxin; signal transduction

\section{INTRODUCTION}

Meiosis and subsequent spermatogenesis are dynamic processes whereby marked changes occur in the chromatin structure and cellular morphology of the testes. DNA-binding proteins, histones, are converted to protamines via transition proteins. ${ }^{1}$ During this process, the DNA is exposed to a deleterious environment, which includes oxidative stress. To transfer superior genes to offspring, germ cells are protected under rigid quality control, and rejected cells are mostly eliminated. Enzyme-mediated oxidative crosslinking between protamines occurs and is involved in the compaction of nuclear substances. This process, known as sperm maturation, occurs in the epididymidis. However, oxidation often causes harmful conditions, called oxidative stress, which results in oxidative damage to the DNA.

Reactive oxygen species (ROS) are molecules directly involved in the oxidation process, that is, the condition in which ROS and prooxidants predominate over antioxidants. ROS are produced during vital activities, such as oxygen consumption, by the mitochondrial electron transport system and under various stress conditions, and appear to be involved in such cytotoxic processes as heat stress and ischemia of the testes. ${ }^{2-4}$ Oxidative stress decreases sperm motility and the ability to fuse with the oocyte. Spermatogenic cells with DNA damage are prone to elimination by apoptosis via p53-dependent and -independent mechanisms. ${ }^{5,6}$ Thus, male infertility is often attributable to oxidative stress. ${ }^{7}$
Regarding ROS-mediated reactions, the signalling role of ROS in the proliferation of somatic cells has attracted considerable attention during the last two decades. ${ }^{8}$ Enzymes that possess a highly reactive cysteine (Cys) in either the catalytic centre or a regulatory site play a role in the transmission of ROS signals. Tyrosine phosphorylation/ dephosphorylation in response to extracellular stimuli has been characterized most thoroughly, but only a limited number of biological processes, such as signalling in response to mitotic stimuli, are known. ${ }^{9}$

Here, we reviewed recent advances in the understanding of redox systems as a defence in the testes and in the fertilisation process; we also reviewed a regulatory mechanism in cellular signal transduction and the reproductive process that involves redox reactions.

\section{ROLES OF SULPHOXIDATION IN SPERMATOGENESIS}

Spermatogonia continue to proliferate after sexual maturation, and the resulting spermatogenic cells differentiate to sperm. After meiosis, spermatogenic cells undergo tremendous morphological changes as they develop into sperm. During this process, the histones in chromatin are converted to transition proteins and finally to spermspecific histones, collectively referred to as protamines, in sperm. While the primary structure of the histones is conserved among different species, protamine is highly variable, which is essential for individual species' survival by preventing fertilisation by other species. ${ }^{10}$ 
The amino acid that differs most strikingly between histones and protamines is Cys. While only one Cys is present in each of the histones, it is an abundant amino acid in the protamines of mammalian sperm. Disulfhydryl formation, due to the oxidation of nearby sulphydryls, contributes to the packaging of chromatin and a decrease in nucleus volume (Figure 1). This oxidative packaging of chromatin protects the sperm head from impairment, including physical damage and DNA oxidation, and enables rapid movement during the fertilisation process. ${ }^{11}$ Sulphoxidation mediated by specific enzymes found in the epididymidis causes the oxidative packaging of nucleoproteins during the sperm maturation process. Notably, the quality of sperm can be evaluated using reagents that distinguish sulfhydryl from disulfhydryl; the higher the disulfhydryl bridge content, the better the quality of the sperm. ${ }^{12}$ Thus, regulated disulfhydryl bond formation in proteins contributes to the maintenance of the high viability of sperm. $^{13}$

However, exposure of DNA to ROS causes oxidative modification of bases, base substitution and DNA strand break, ${ }^{12}$ which transmits adverse genetic information to offspring. In the case of lipids, polyunsaturated fatty acids are the preferential target of ROS. In human sperm, approximately $40 \mathrm{wt}-\%$ of the total fatty acid fraction is composed of polyunsaturated fatty acids, which are vulnerable to peroxidation by ROS. ${ }^{14}$ Because peroxidized lipids and the resulting carbonyl compounds suppress sperm motility, protection against oxidative stress improves the fertility of sperm. ${ }^{15}$ Thus, oxidation affects sperm in complex ways, depending on the physiological conditions of the sperm and environmental factors, notably oxygen and antioxidants. $^{16,17}$

\section{REGULATION OF THE INTRACELLULAR SIGNAL MEDIATED BY ROS}

With respect to the signal-modulating function of ROS, most work has been performed on somatic cells under culture conditions. Data from in vivo studies are gradually accumulating, indicating that the same mechanism may also be applicable for reproductive systems. A consensus mechanism for the signalling function of ROS is summarized in Figure 2. Tyrosine kinase-type receptors are commonly involved in intracellular signal transduction of growth factors and cytokines. It is generally understood that the extracellular signal attributed to tyrosine kinase-type receptor is mediated by autophosphorylation and transmitted to subsequent molecules including adaptors, $G$ proteins and

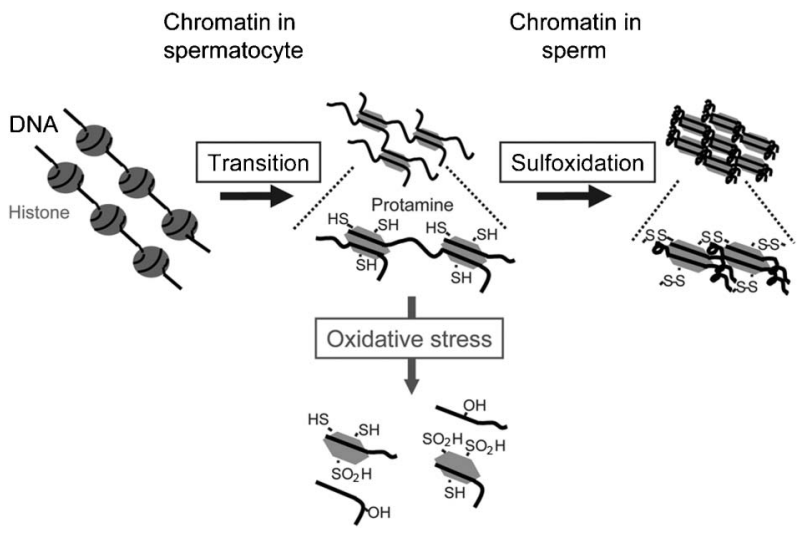

Figure 1 Chromatin structures in spermatocyte and sperm in normal spermatogenesis and under oxidative stress. $\mathrm{SH}$, cysteine sulphydryl; $\mathrm{SOH}$, cysteine sulphenic acid; $\mathrm{SO}_{2} \mathrm{H}$, cysteine sulphinic acid.

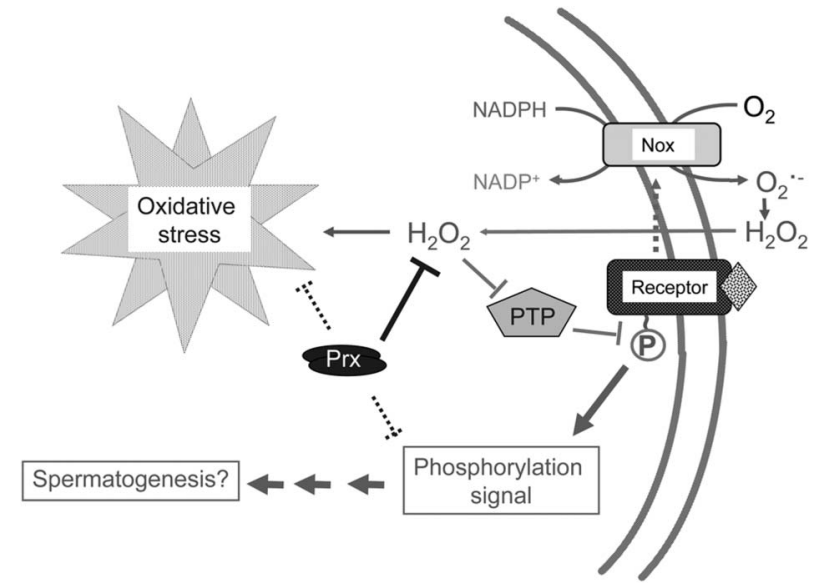

Figure 2 Schematic presentation of hydrogen peroxide-mediated regulation of phosphorylation signals in response to extracellular stimuli. Nox, NADPHdependent oxidase; Prx, peroxiredoxin; PTP, phosphotyrosine phosphatase.

effector molecules. Each kinase reaction is associated with a phosphatase to stop the signal mediated by phosphorylation.

It has been known for some time that ROS are concomitantly produced in response to mitotic stimuli, but the physiological significance of the ROS has been elucidated only recently. The newly identified role of ROS, mainly hydrogen peroxide, is the oxidative inactivation of the catalytic centre of certain phosphatases. ${ }^{8} \mathrm{PTP} 1 \mathrm{~B}$, a phosphotyrosine phosphatase, and PTEN, a phosphatase for phosphatidyl inositide, are inactivated by hydrogen peroxide that is generated concomitantly with activation of the phosphorylation signal. ${ }^{18-20}$ NADPH oxidase appears to be involved in the modulation of this signalling process by producing superoxide via one-electron donation to molecular oxygen, consequently generating hydrogen peroxide through the spontaneous dismutation of superoxide. While superoxide is produced outside cells and cannot penetrate the lipid bilayer, hydrogen peroxide can permeate the cells and oxidatively inactivate phosphatases that possess highly reactive cysteine sulphydryl (Cys-SH) at the catalytic centre. The oxidative modification of Cys-SH to $\mathrm{Cys}-\mathrm{SOH}$ is reversible; hence, the inactivated phosphatase recovers the activity and stops the signal by dephosphorylation, because sustained inactivation of the phosphatases may cause continuous cellular proliferation and, consequently, tumorigenic proliferation of cells. The tumour suppressor function of PTEN can thus be maintained. Meanwhile, conditional knockout of PTEN in oocytes causes premature activation of the primordial follicle in mice by sustained activation of the PI3kinase/Akt system. ${ }^{21,22}$ This suggests that, in the primordial follicle, PTEN is constitutively active and keeps the cellular state in the follicle on standby. While kinases and phosphatases are closely connected, ROS regulate the balance of their activity by oxidatively modifying active site Cys in the phosphatases. A similar mechanism is present in most cells and regulates cellular functions.

\section{PEROXIREDOXIN (PRX) AS A PIVOTAL CONTROLLER OF THE ROS SIGNAL}

Elimination of excess ROS is required for effective extracellular signal function. This surveillance function appears to be fulfilled by Prx, a new family of redox proteins identified fairly recently. The Prx family is classified into six distinct gene products in mammals. ${ }^{23}$ There are three Prx subtypes: four are members of the typical 2-Cys Prx subtype, one belongs to the atypical 2-Cys Prx subtype and one belongs to the 
1-Cys Prx subtype. Thioredoxin-dependent peroxidase activity was the first type to be reported and is common to most Prx family members. This peroxidase activity appears to be responsible for the regulatory function of ROS signalling.

The most extensively characterized members are Prx1 and Prx2, which predominate in many cells. One of their major functions appears to be the protection of phosphatases from inactivation by eliminating excess hydrogen peroxide in cells responding to growth factors. ${ }^{8}$ The presence of some Prx members in the testes is confirmed, but the roles of Prx in the male reproductive system and sperm are still obscure. Among the isoforms, Prx4 exhibits complex characteristics compared to the other Prx family members. A typical $\mathrm{N}$-terminal signal sequence for secretion is found in Prx4, which implies a role of proteins in the extracellular milieu. ${ }^{24,25}$ A possible role in the testes has been implied, because expression is the highest in the testes, and there are two forms that differ in size. A small, ordinary form is present in most tissues, and a large form is present mainly in sexually maturing testes. ${ }^{26}$ The size of the small form corresponds to a processed polypeptide that lacks the N-terminal signal sequence and is retained in the Golgi body by forming a homodecamer and/or secreted to the extracellular milieu. ${ }^{25,27} \mathrm{~A}$ pivotal role for protection by $\operatorname{Prx} 4$ at a specific stage of spermatogenesis is supported by a previous report demonstrating that experimentally induced cryptorchidism causes damage in germ cells at the stage when the high-molecular weight form of Prx4 is expressed. ${ }^{28}$ Thus, Prx4 in the testes appears to protect spermatogenic cells against damage by ROS, while the small Prx4 exerts its antioxidant role in the endoplasmic reticulum (ER)/Golgi body.

Prx4-knockout mice have been generated, and preliminary analysis of their phenotypes has been reported. ${ }^{29}$ A prominent knockout phenotype was observed mainly in the testes, where the highmolecular weight form of Prx4 is present in sexually mature wild-type male mice. An abnormally high number of spermatogenic cells died, even under specific pathogen-free breeding conditions, which were further deteriorated by brief heat stress. This is in sharp contrast with other Prx members, because no abnormal phenotypes have been reported in the testes of mice that lack Prx1, Prx 2 or Prx3. Because the elevation of lipid peroxidation reaction products and the oxidation of protein sulphydryls and DNA were observed in Prx4-deficient mouse testes, the abnormal cell death is likely attributable to elevated oxidative stress due to Prxy deficiency in spermatogenic cells. Although ROS are inevitably produced during oxygen consumption in the mitochondria, where more than $90 \%$ of respired oxygen is consumed, some oxygen is also consumed by other enzymatic reactions, such as the P450 system in the ER. Hydrogen peroxide is reportedly produced at high levels by the reaction of Ero-1 in the lumen of the ER. ${ }^{30}$ Thus, the localisation of Prx 4 may imply an essential role in the detoxification of hydrogen peroxide in the ER. In fact, when B cells are differentiated or stimulated by lipopolysaccharide, the expression of Prx4 is enhanced. ${ }^{31}$ Among various stresses, elevated ROS causes sulphydryl oxidation and resulting disulfhydryl bond formation in the ER. Because Prx4 is located in the ER and has protein disulfhydryl isomerase activity, Prx4 may catalyse a protein disulfhydryl isomeraselike reaction to recruit the proteins misfolded due to formation of the wrong disulfhydryl bond. ${ }^{27}$ Accumulating evidence suggests that the ER regulates intracellular calcium levels and apoptosis in some cells. There may be a link between calcium homeostasis and Prx4 function in ER/Golgi bodies. In addition to Prx4, the presence of Prx2 in sperm has been demonstrated, although the physiological role of Prx2 is not clear. $^{32}$

\section{REDOX REGULATION OF SPERM FUNCTION}

The deteriorating effects of the oxidation reaction in sperm and the possible treatment of infertile male patients with antioxidants have been repeatedly reviewed. ${ }^{3,33-35}$ Although spermatogenic cells are protected by many antioxidative and redox systems to support normal spermatogenesis, sperm has a very low capacity with respect to cytoplasmic volume; hence, it possesses only a limited number of protective systems. The presence and oxidative modification of Prx1, Prx4, Prx5 and Prx6 by hydrogen peroxide suggests their protective roles against oxidative stress. ${ }^{36}$ In addition, low levels of ROS are essential for sperm functions such as capacitation and activation, and sperm indeed produces ROS., ${ }^{3,37}$ In vivo, the lining fluids in the female reproductive tract provide protective agents such as antioxidants and reductants, but during the in vitro fertilisation process, antioxidants supplemented in the incubation medium may support sperm function.

Among millions of sperm ejaculated into the female reproductive tract during coitus, only a small number reach the ampulla, the site of fertilisation. Sperm transiently adhere to the epithelial cells of the lower region of the oviduct. The fertile life span of sperm is extended by delaying capacitation. Interactions between carbohydrates on the oviduct cells and lectin-like molecules on the sperm surface appear to be involved in this adhesion. ${ }^{37}$ Although the precise nature of the molecules responsible for this process has not yet been identified, the redox status of the sperm surface appears to be a determining factor in whether a sperm adheres or releases to this oviduct because the reductants affect sperm adhesion. ${ }^{38,39}$ Among the disulfhydryl reductants, glutathione is a promising candidate in the female reproductive tract because glutathione is predominantly present and recycled by glutathione reductase, which is plentiful in the epithelial cells of the oviduct and uterus. ${ }^{40}$ The sperm adhesion process is species-specific, but most evidence comes from pigs and cattle. Thus, analyses of human sperm and epithelial cells are required to understand the mechanisms and to apply the results for clinical purposes.

\section{PERSPECTIVES}

It is now a common view that oxidative stress is deeply involved in testicular dysfunction, sperm damage, and, as a consequence, male infertility. Administration of antioxidants, as medicine or supplements, appears to be beneficial for the maintenance of male fertility. Recent advances in redox biology are unveiling the novel advantageous roles of ROS in cell signalling functions. Because excess intake of antioxidants may affect the signalling role of ROS and may thereby impair testicular function, coordinated studies from both basic and clinical viewpoints would provide clues helpful in resolving seemingly conflicting phenomena concerning male infertility.

\section{COMPETING FINANCIAL INTERESTS}

The authors declare no competing financial interests.

\section{ACKNOWLEDGMENTS}

This work was partly supported by a grant for Research on Child Health and Development from the Ministry of Health, Labor and Welfare, Japan, and by the YU-COE Program at Yamagata University.

1 Erenpreiss J, Spano M, Erenpreisa J, Bungum M, Giwercman A. Sperm chromatin structure and male fertility: biological and clinical aspects. Asian J Androl 2006; 8: 11-29. 
2 Aitken RJ, Baker MA. Oxidative stress, sperm survival and fertility control. Mol Cell Endocrinol 2006; 250: 66-9.

3 Agarwal A, Makker K, Sharma R. Clinical relevance of oxidative stress in male factor infertility: an update. Am J Reprod Immunol 2008; 59: 2-11.

4 Tremellen K. Oxidative stress and male infertility-a clinical perspective. Hum Reprod Update 2008; 14: 243-58.

5 Yin Y, DeWolf WC, Morgentaler A. Experimental cryptorchidism induces testicular germ cell apoptosis by p53-dependent and-independent pathways in mice. Biol Reprod 1998; 58: 492-6.

6 Ohta $\mathrm{H}$, Aizawa S, Nishimune Y. Functional analysis of the p53 gene in apoptosis induced by heat stress or loss of stem cell factor signaling in mouse male germ cells. Biol Reprod 2003; 68: 2249-54.

7 Garrido N, Meseguer M, Simon C, Pellicer A, Remohi J. Pro-oxidative and antioxidative imbalance in human semen and its relation with male fertility. Asian $J$ Androl 2004; 6: 59-65.

8 Rhee SG, Kang SW, Jeong W, Chang TS, Yang KS et al. Intracellular messenger function of hydrogen peroxide and its regulation by peroxiredoxins. Curr Opin Cell Biol 2005; 17: 183-9.

9 Cho $\mathrm{SH}$, Lee $\mathrm{CH}$, Ahn Y, Kim H, Kim H et al. Redox regulation of PTEN and protein tyrosine phosphatases in $\mathrm{H}_{2} \mathrm{O}_{2}$ mediated cell signaling. FEBS Lett 2004; 560: 7-13.

10 Wyckoff GJ, Wang W, Wu Cl. Rapid evolution of male reproductive genes in the descent of man. Nature 2000; 403: 304-9.

11 Pfeifer H, Conrad M, Roethlein D, Kyriakopoulos A, Brielmeier M et al. Identification of a specific sperm nuclei selenoenzyme necessary for protamine thiol cross-linking during sperm maturation. FASEB J 2001; 15: 1236-8.

12 Andrabi SM. Mammalian sperm chromatin structure and assessment of DNA fragmentation. J Assist Reprod Genet 2007; 4: 61-9.

13 Sharma RK, Agarwal A. Role of reactive oxygen species in male infertility. Urology 1996; 48: 835-50.

14 Wathes DC, Abayasekara DR, Aitken RJ. Polyunsaturated fatty acids in male and female reproduction. Biol Reprod 2007; 77: 190-201.

15 Sanocka D, Kurpisz M. Reactive oxygen species and sperm cells. Reprod Biol Endocrinol 2004: 2: 12-8.

16 Aitken RJ, Fisher H. Reactive oxygen species generation and human spermatozoa: the balance of benefit and risk. Bioessays 1994; 16: 259-67.

17 Aitken RJ, Ryan AL, Baker MA, McLaughlin EA. Redox activity associated with the maturation and capacitation of mammalian spermatozoa. Free Radic Biol Med 2004 36: 994-1010.

18 Lee SR, Kwon KS, Kim SR, Rhee SG. Reversible inactivation of protein-tyrosine phosphatase $1 \mathrm{~B}$ in A431 cells stimulated with epidermal growth factor. J Biol Chem 1998; 273: 15366-72.

19 Lee SR, Yang KS, Kwon J, Lee C, Jeong W et al. Reversible inactivation of the tumor suppressor PTEN by $\mathrm{H}_{2} \mathrm{O}_{2}$. J Biol Chem 2002; 277: 20336-42.

20 Leslie NR, Bennett D, Lindsay YE, Stewart $\mathrm{H}$, Gray A et al. Redox regulation of $\mathrm{PI}$ 3-kinase signalling via inactivation of PTEN. EMBO J 2003; 22: 5501-10.

21 Reddy P, Liu L, Adhikari D, Jagarlamudi K, Rajareddy S et al. Oocyte-specific deletion of Pten causes premature activation of the primordial follicle pool. Science 2008; 319: 611-3.
22 John GB, Gallardo TD, Shirley LJ, Castrillon DH. Foxo3 is a PI3K-dependent molecular switch controlling the initiation of oocyte growth. Dev Biol 2008; 321: 197-204.

23 Fujii J, Ikeda Y. Advances in our understanding of peroxiredoxin, a multifunctional, mammalian redox protein. Redox Rep 2002; 7: 123-30.

24 Matsumoto A, Okado A, Fujii T, Fujii J, Egashira M et al. Cloning of the peroxiredoxin gene family in rats and characterization of the fourth member. FEBS Lett 1999; 443 246-50.

25 Okado-Matsumoto A, Matsumoto A, Fujii J, Taniguchi N. Peroxiredoxin IV is a secretable protein with heparin-binding properties under reduced conditions. J Biochem 2000; 127: 493-501.

26 Sasagawa I, Matsuki S, Suzuki Y, luchi Y, Tohya K et al. Possible involvement of the membrane-bound form of peroxiredoxin 4 in acrosome formation during spermiogenesis of rats. Eur J Biochem 2001; 268: 3053-61.

27 Tavender TJ, Sheppard AM, Bulleid NJ. Peroxiredoxin IV is an endoplasmic reticulumlocalized enzyme forming oligomeric complexes in human cells. Biochem J 2008; 411: 191-9.

28 Matsuki S, Sasagawa I, luchi Y, Fujii J. Impaired expression of peroxiredoxin 4 in damaged testes by artificial cryptorchidism. Redox Rep 2002; 7: 276-8.

29 Iuchi Y, Okada F, Tsunoda S, Kibe N, Shirasawa N et al. Peroxiredoxin 4 knockou results in elevated spermatogenic cell death via oxidative stress. Biochem J 2009, 419: 149-58.

30 Enyedi B, Varnai P, Geiszt M. Redox state of the endoplasmic reticulum is controlled by Ero1L-alpha and intraluminal calcium. Antioxid Redox Signal 2010; 13: 721-9.

31 Bertolotti M, Yim SH, Garcia-Manteiga JM, Masciarelli S, Kim YJ et al. B- to plasmacell terminal differentiation entails oxidative stress and profound reshaping of the antioxidant responses. Antioxid Redox Signal 2010; 13: 1133-44.

32 Manandhar G, Miranda-Vizuete A, Pedrajas JR, Krause WJ, Zimmerman S et al. Peroxiredoxin 2 and peroxidase enzymatic activity of mammalian bpermatozoa. Biol Reprod 2009; 80: 1168-77.

33 Lanzafame FM, La Vignera S, Vicari E, Calogero AE. Oxidative stress and medical antioxidant treatment in male infertility. Reprod Biomed Online 2009; 19: 638-59.

34 Kefer JC, Agarwal A, Sabanegh E. Role of antioxidants in the treatment of male infertility. Int J Urol 2009; 16: 449-57.

35 Zini A, San Gabriel M, Baazeem A. Antioxidants and sperm DNA damage: a clinical perspective. J Assist Reprod Genet 2009; 26: 427-32.

36 O'Flaherty C, Rico de Souza A. Hydrogen peroxide modifies human sperm peroxiredoxins in a dose-dependent manner. Biol Reprod 2010; 84: 238-47.

37 Talevi R, Gualtieri R. Molecules involved in sperm-oviduct adhesion and release. Theriogenology 2010; 73: 796-801.

38 Talevi R, Zagami M, Castaldo M, Gualtieri R. Redox regulation of sperm surface thiols modulates adhesion to the fallopian tube epithelium. Biol Reprod 2007; 76: 728-35

39 Gualtieri R, Mollo V, Duma G, Talevi R. Redox control of surface protein sulphhydryls in bovine spermatozoa reversibly modulates sperm adhesion to the oviductal epithelium and capacitation. Reproduction 2009; 138: 33-43.

40 Kaneko T, luchi Y, Kawachiya S, Fujii T, Saito H et al. Alteration of glutathione reductase expression in the female reproductive organs during the estrous cycle. Biol Reprod 2001; 65: 1410-6. 